\title{
Trismus- An Old Concept For Successfull Treatment in The Present Era - Case Reports
}

\author{
Prof.Dr Amit Ray ${ }^{1}$. Dr Partha Sundar Biswas ${ }^{2}$ Dr Abhisek Chatterjee ${ }^{3}$ \\ ${ }^{\prime}$ professor \& Head Of The Department Of Oral \& Maxillofacial Surgery, Guru Nanak Institute Of Dental \\ Scoience\& Research,Panihati, Kolkata, West Bengal \\ ${ }^{2 \& 3} 3^{\text {rd }}$ Year Pgt, Department Of Oral \& Maxillofacial Surgery, Guru Nanak Institute Of Dental Scoiences \& \\ Research ,Panihati, Kolkata, West Bengal
}

\begin{abstract}
Trismus, a tonic contraction of the jaw-closing muscles, has now received broader application in use, including all conditions characterized by the inability to open the mouth adequately. There are so many pathological conditions which causes trismus. One of the main cause is inflammation of masticatory muscle due to infection and trauma. Historically Esmarch was first one to perform osteotomy for TMJ ankylosis in 1851. A flap of masseter and medial pterygoid sutured and interposed between a wedge shaped osteotomy cut at angle of mandible and will act as a pseudojoint.
\end{abstract}

\section{Introduction}

${ }^{5}$ Trismus may be defined as inability to open the mouth due to muscle spasm, but the term is usually used for limited movement of the jaw from any cause. The normal range of mouth opening varies from patient to patient, within a range of $40-60 \mathrm{~mm}$, although some authors place the lower limit at $35 \mathrm{~mm}$. The width of the index finger at the nail bed is between 17 and $19 \mathrm{~mm}$. Thus, two fingers' breadth $(40 \mathrm{~mm})$ up to three fingers' breadth $(54-57 \mathrm{~mm})$ is the usual width of opening. Evidence suggests that gender may be a factor in vertical mandibular opening. In general, males display greater mouth opening. Lateral movement is $8-12 \mathrm{~mm}$.

Several conditions may cause or predispose an individual to develop trismus. The aetiology of trismus may be classified as follows:

1. Infection

2. Trauma

3. Dental treatment

4. Temporomandibular joint disorders

5. Tumours and oral care drugs

6. Radiotherapy and chemotherapy

7. Congenital problem

8. Miscellaneous disorders.

Treatment of trismus varies depending on the aetiological factors, ranging from medical to surgical. This paper deals with the treatment of long standing trismus by esmarch procedure. ${ }^{5}$

\section{Historical perspective}

${ }^{1}$ Historically Esmarch was said to be the first one to perform osteotomy for TMJ ankylosis in 1851 whilst Humphrey performed the first condylectomy in 1854. Gap arthroplasty was first advocated by Abbe in 1880 and interpositional material was first used by Risdon in 1934 . As per Esmarch procedure small incision was made on each side behind the angle of the mandible, and the masseter and internal pterygoid muscles were separated from the bone to their most anterior limits of attachment. A wedge of bone,with a base about $\mathbf{2 . 5}$ centimetres (one inch) wide, at posterior border mandible including angle with apex at upper border, was then nibbled away with bone forceps. On removal of the bone, the exposed masseter and medial pterygoid muscles were joined by a few sutures and the wound was closed. 

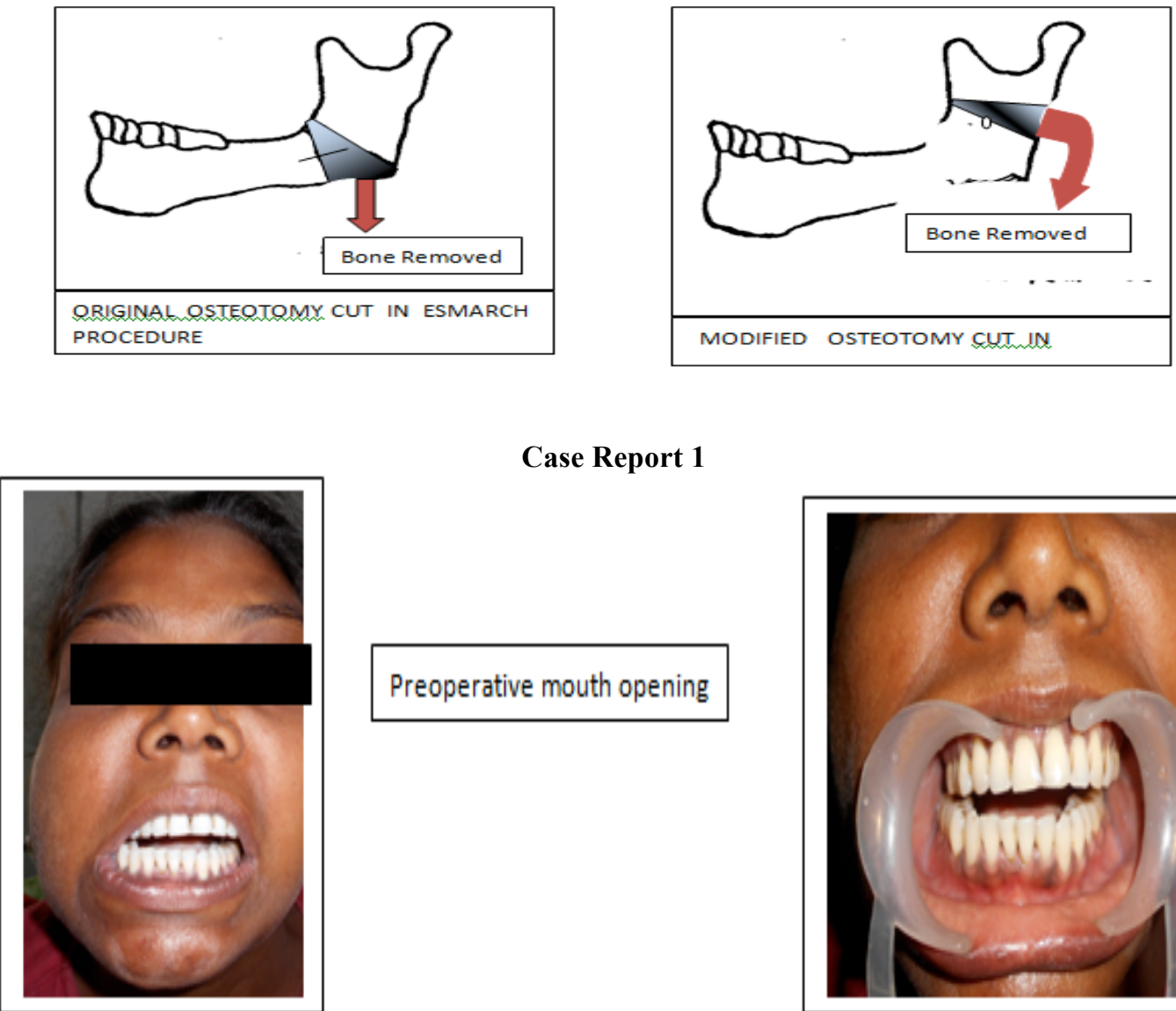

\section{Case Report 1}

Preoperative mouth opening

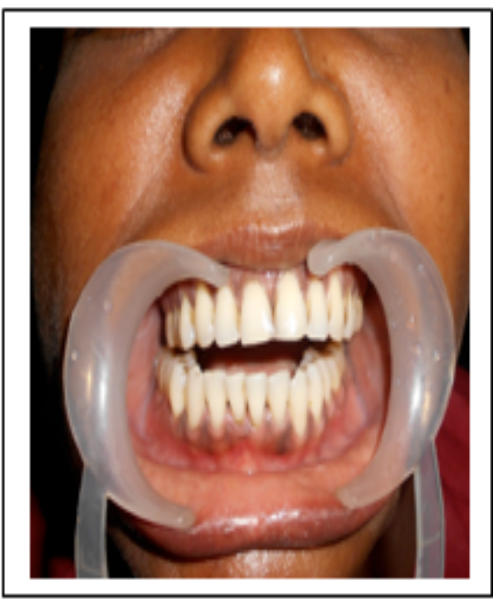

A 36 year female patient came to our department with a complaint of persistent trismus for 2-3 months and swelling at right side of face. History reveals that the patient had a pain at right lower back teeth region 4 months back. She took some medicine for relief of pain but slowly she developed swelling at right side of face. She then consulted with a dental surgeon at Domjur Howrah. Inspite of taking antibiotic and analgesic as per prescription of that doctor, pain and swelling did not subside. Then she went to SSKM \& IPGMER Kolkata at ENT Department and took admission for incision and drainage where the incision and drainage was done thrice in a span of seven days as per diagnosis of buccal space abscess. The swelling subsided subsequently to some extent but after three weeks she again developed trismus.There after she sought treatment at Dr R Ahmed Dental College \& Hospital Kolkata. She was advised an OPG and noncontrast CT scan which revealed a large thick walled heterogenous lesion in right side of cheek involving right masseter and deep part of parotid including infratemporal fossa region.

Thereafter she reported to our department for further treatment. Medical history revealed nothing significant. OPG revealed a radiolucent area in the periapical region of carious right lower first molar. On detailed history taking the patient complained of pain in the tooth (First molar) since 6-8 months which preceded trismus. Subsequently a CECT of face revealed that an enhancing soft tissue lesion involving the right buccal mucosa, retromolar trigone area inferior gingivobuccal sulcus masseter and right submandibular region. Some inflammatory changes and loss of fat plane noted in both infratemporal and submasseteric space. 


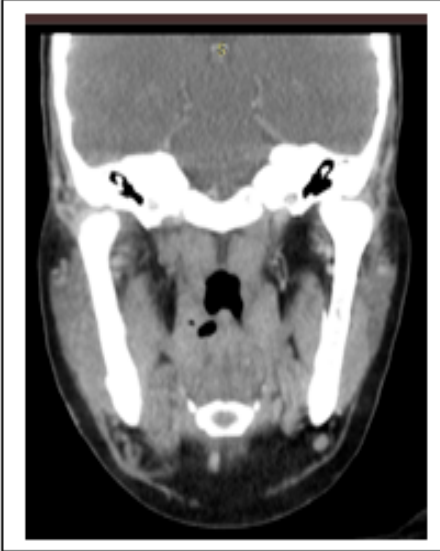

A

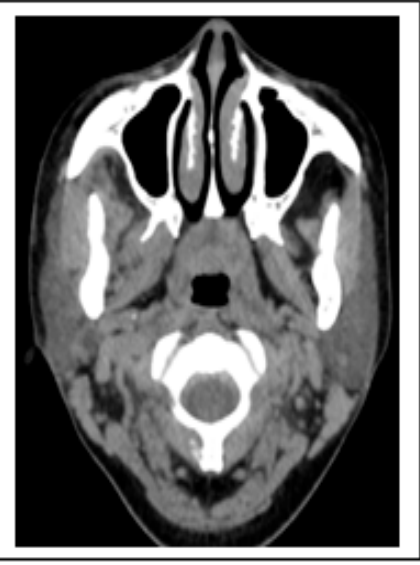

B

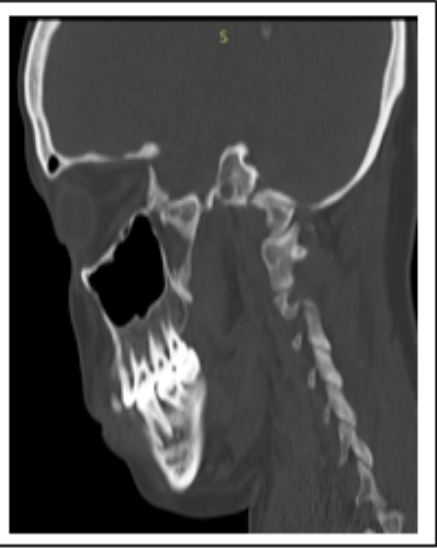

C

\section{CECT of face}

A. Coronal view and B. Axial view shows diffuse inflammatory changes in masseter, medial pterygoid, infratemporal space

C. Sagital view shows periapical lesion in relation to lower first molar

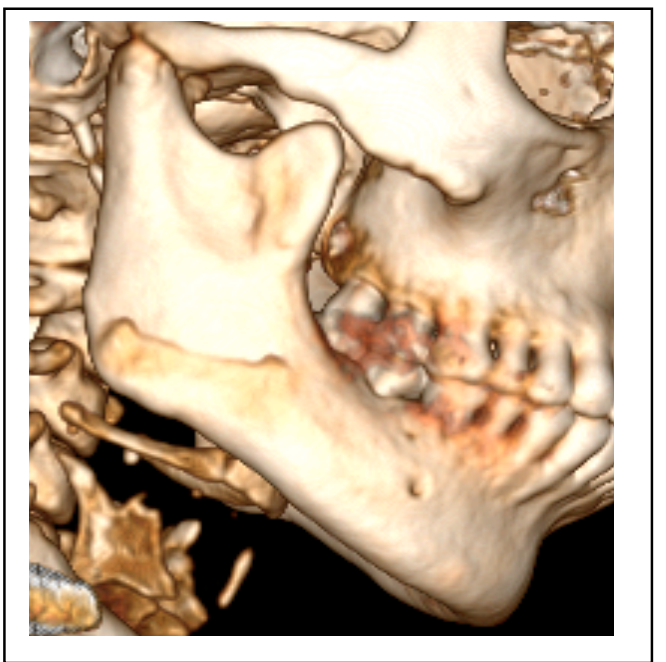

D

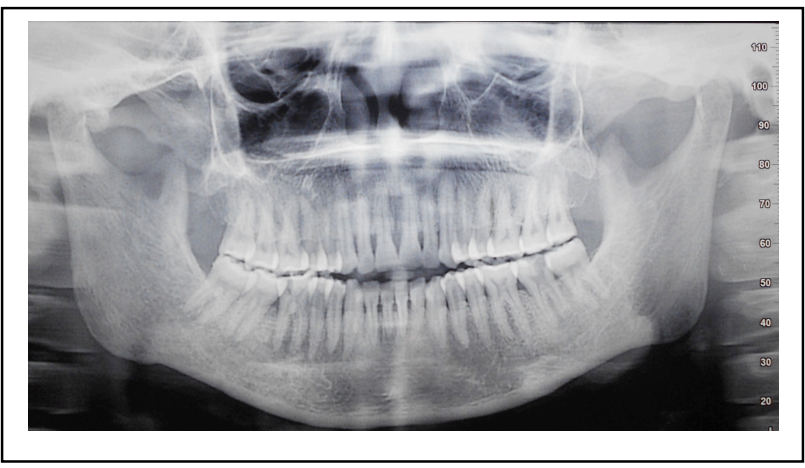

E

D . 3D reconstruction of CECT face shows inflammatory recative bone formation at masseteric notch area.

E. OPG shows periapical radiolucency at lower first molar region.

\section{Surgical Procedure}

Esmarchs operation was planned for releasing trismus and early active jaw exercise .Patient was anesthetized by fiberoptic intubation. Body of mandible was exposed by submandibular incision and inflammatory reactive subperiosteal bone was removed from region at body of mandible. After marking an wedge shaped bone above the level of lingual medial pterygoid and masseter muscles are sutured by 2-0 vicryl which was act as a pseudojoint. Closure done by3-0 vicryl and surgical stapler. 

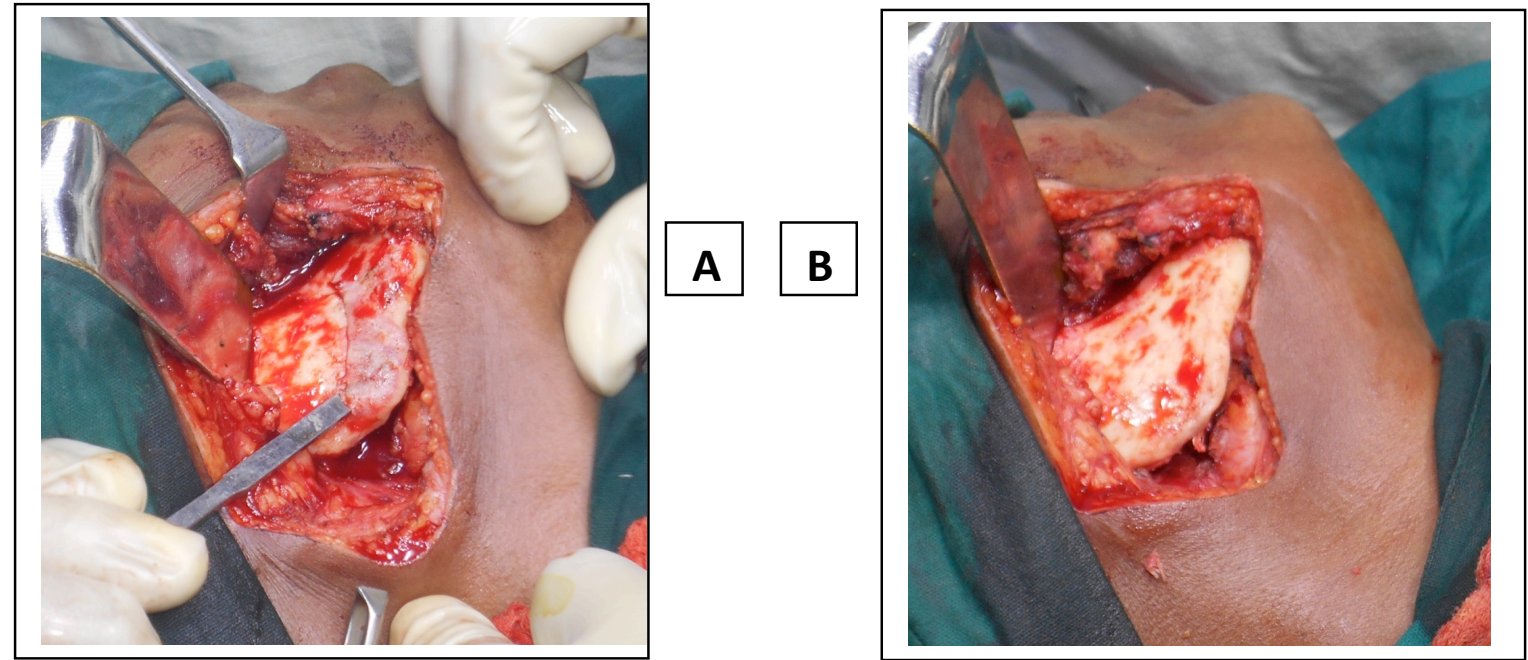

Surgical procedure:- A-After exposing body and ramus of the mandible,subperiosteal reactive bone removed with the help of osteotomy, B- Following removal of reactive bone.

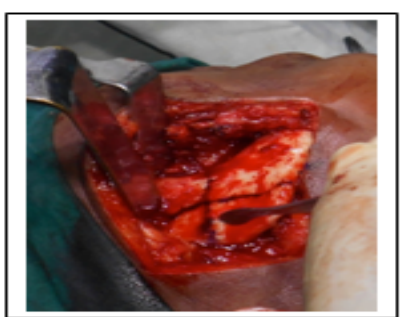

c

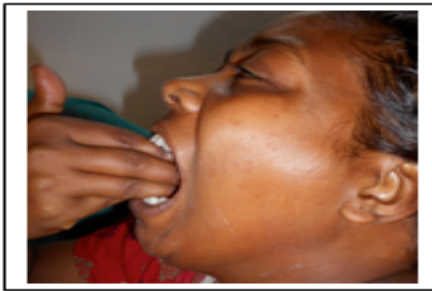

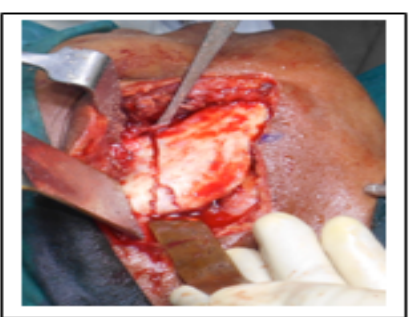

D

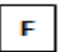

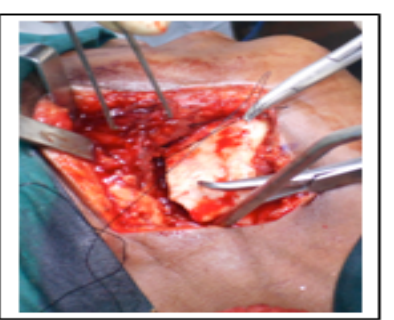

E

$\mathbf{G}$

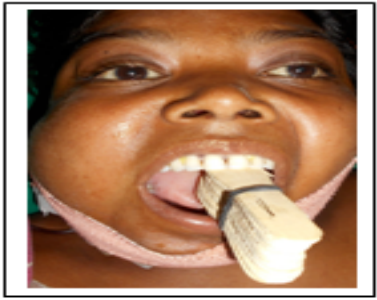

C- Marking antilingula D- Osteotomy cut above the lingual E- Suturing of both masseter and medial pterygoid muscle to act as pseudojoint $\mathbf{F}$ - After $3^{\text {rd }}$ post operative day mouth opening G- Active mouth exercise by bunch of icecream sticks.

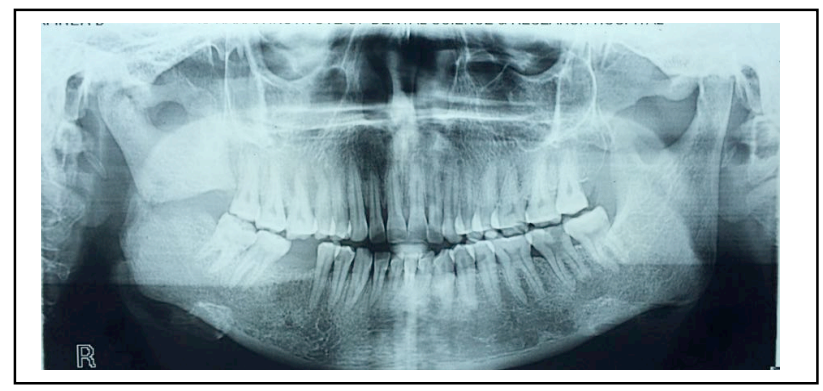

Post operative OPG after

4 month 


\section{Case No 2}

A patient of 70 years age, with history of COPD and hypertension was admitted in our hospital on 31/1/2014 with bilateral high subcondylar fracture along with displacement of both condyles in middle cranial fossa resulting in absence of mandibular movements..In this case also an Esmarch operation was done with favourable results.
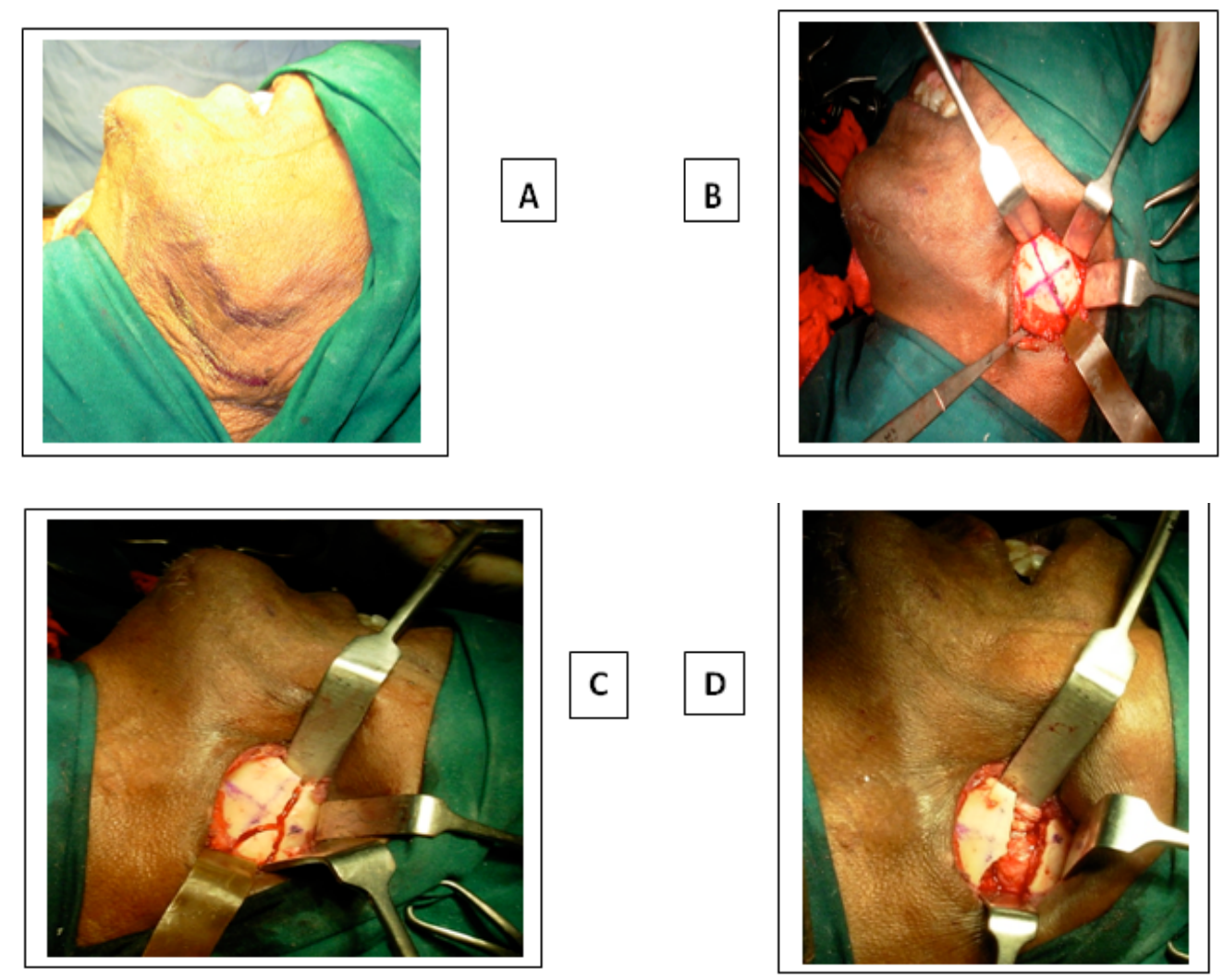

A:-Marking for submandibular incision at right side.

B:-Marking for antilingula.

C:- Marking for horizontal osteotomy cut .

D:- After completion of osteotomy and a part of medial pterygoid.

\section{Conclusion}

As mentioned previously, trismus is a clinical condition with variable etiology. To understand the etiology is of primr importance in treatment of the same. There are some situation where lack of early requisite treatment may result in intence fibrosis of the closing muscles of the mandible as well as some fractures of proximal part of the mandible which might result in diminished mandibular movements. The procedure described by Esmarch way back in 1851 still holds good in the present era in some cases as depicted in this article .

It has been our experience that this procedure is a very simple surgery associated with no long term complications yet providing with a excellent result as far as restoring normal mandibular function. There was no significant malocclusion in both the cases on long term follow up. Thus this procedure can be judiciously used in cases with long standing trsmus depending upon the etiological factors.

\section{References}

[1]. Ankylosis of temporomandibular joint : cure by Esmarch's operatrion

[2]. by I Douglus Miller,Sydney.

[3]. Management of Temporomandibular joint ankylosis:Literature review,Zubair Khatun FRCS,FFDRCS(Pakistan Oral and Dent. Jr.25(2) Dec 2005

[4]. Limited painfull mouth opening :Nurhan Guler,DDS Phd,Perran Fulden Yumuk,MD,Dilhun Ilguy DDS Phd,Vakur Olgak MD,Jerry Greer DDS ( JOMS 63:1201-1205,2005)

[5]. Surgical pathology of the mouth and jaws: R A Cawson,J D Langdon,J W Eveson.

[6]. Trismus :Etiology differential diagnosis and treatment - P J Dhanrajani and O Jonaidel. 
